\title{
Science with the Keck Interferometer ASTRA Program
}

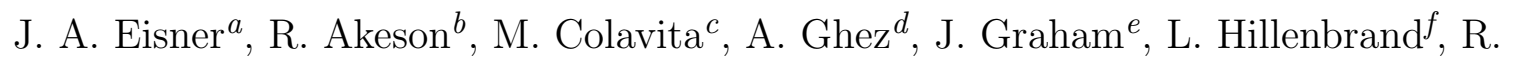 \\ Millan-Gabet ${ }^{b}$, J.D. Monnier ${ }^{g}$, J.-U. Pott ${ }^{h}$, S. Ragland ${ }^{i}$, P. Wizinowich ${ }^{i}$, J. Woillez ${ }^{i}$ \\ ${ }^{a}$ Steward Observatory, University of Arizona, Tucson, AZ 85721 \\ ${ }^{b}$ NASA Exoplanet Science Institute, Caltech, Pasadena, CA 91125 \\ ${ }^{c}$ Jet Propulsion Laboratory, Caltech, Pasadena, CA 91109 \\ ${ }^{d}$ Astronomy Department, UCLA, Los Angeles, CA 90095 \\ ${ }^{e}$ Astronomy Department, UC Berkeley, Berkeley, CA 94720 \\ ${ }^{f}$ Astrophysics Department, Caltech, Pasadena, CA 91125 \\ ${ }^{g}$ Astronomy Department, University of Michigan, Ann Arbor, MI 48109 \\ ${ }^{h}$ Max-Planck-Institut für Astronomie, Königstuhl 17, D-69117 Heidelberg, Germany \\ ${ }^{i}$ W.M. Keck Observatory, Kamuela, HI 96743
}

\begin{abstract}
The ASTrometric and phase-Referenced Astronomy (ASTRA) project will provide phase referencing and astrometric observations at the Keck Interferometer, leading to enhanced sensitivity and the ability to monitor orbits at an accuracy level of 30-100 microarcseconds. Here we discuss recent scientific results from ASTRA, and describe new scientific programs that will begin in 2010-2011. We begin with results from the "self phase referencing" (SPR) mode of ASTRA, which uses continuum light to correct atmospheric phase variations and produce a phase-stabilized channel for spectroscopy. We have observed a number of protoplanetary disks using SPR and a grism providing a spectral dispersion of $\sim 2000$. In our data we spatially resolve emission from dust as well as gas. Hydrogen line emission is spectrally resolved, allowing differential phase measurements across the emission line that constrain the relative centroids of different velocity components at the 10 microarcsecond level. In the upcoming year, we will begin dual-field phase referencing (DFPR) measurements of the Galactic Center and a number of exoplanet systems. These observations will, in part, serve as precursors to astrometric monitoring of stellar orbits in the Galactic Center and stellar wobbles of exoplanet host stars. We describe the design of several scientific investigations capitalizing on the upcoming phase-referencing and astrometric capabilities of ASTRA.
\end{abstract}

Keywords: Interferometry

\section{INTRODUCTION}

ASTrometric and phase-Referenced Astronomy (ASTRA) is a program of upgrades to the Keck Interferometer funded by an NSF MRI grant. ASTRA combines the power of laser guide star adaptive optics (LGS-AO) on the twin 10-m Keck apertures with dual-field phase referencing (DFPR) to enable interferometric observations of stars fainter than $m_{K}=14$. Precise measurement and monitoring of the baseline separating the two Keck apertures allows astrometric observations with an accuracy of $<50 \mu$ as. These capabilities enable a broad range of new science, and we describe ongoing and upcoming investigations below.

\section{ASTRA CAPABILITIES}

Dual-field phase referencing is analogous to natural guide star AO, in that it uses light from a bright reference star to correct the atmosphere and allow longer integrations. ASTRA uses the light from the reference star to measure the atmospheric phase, and a servo loop then removes these phase motions from a second beam train. A fainter source can be observed with longer integration times using this phase-stabilized second beam train. A sketch of the dual-star setup used by ASTRA is shown in Figure 1.

As a precursor to DFPR, we implemented a simple setup called "self phase-referencing" (SPR). Instead of a faint science target and a bright reference star, we split the light from a single, bright star into a continuum

Optical and Infrared Interferometry II, edited by William C. Danchi, Françoise Delplancke, Jayadev K. Rajagopal, Proc. of SPIE Vol. 7734, 773411 - @ 2010 SPIE · CCC code: 0277-786X/10/\$18 · doi: 10.1117/12.856418 


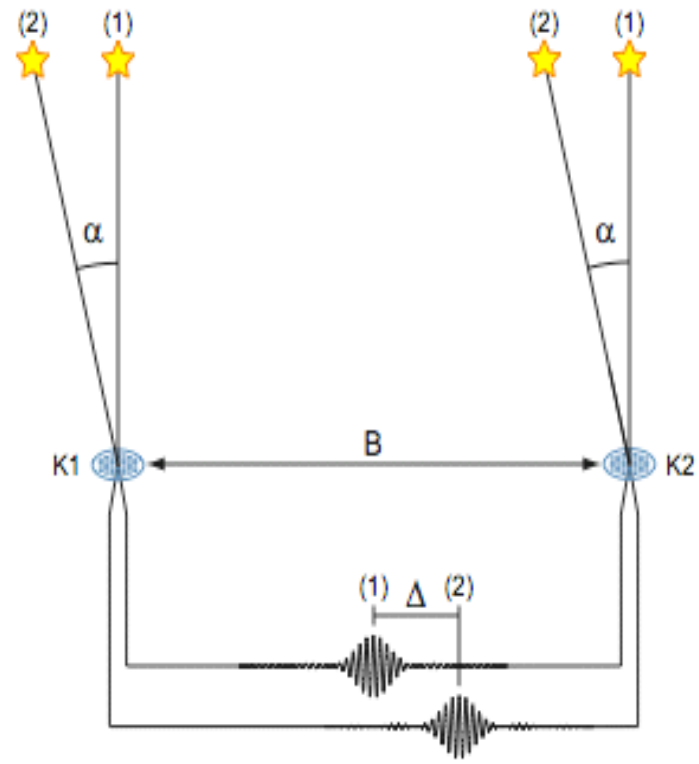

Figure 1. A sketch illustrating the principle of interferometric phase-referencing. The sketch depicts a "dual-star" setup where light from a bright reference star is used to measure atmospheric phase as a function of time. Light from a fainter science target is sent down a separate beam-train that is phase-stabilized using the information from the reference channel. This setup allows longer integrations, and hence greater sensitivity, for the science target. SPR uses a simplified setup, where the reference and science targets are actually the same object. Continuum light from a source is used to correct the atmosphere, and spectrallydispersed light is sent down a phase-stabilized beam-train.

channel and a spectrally dispersed channel. Since all of the light is on-axis in this case, we can split the light near to the beam combination optics, minimizing the non-common path between the two beamtrains. Spectrally dispersed fringes can be observed in the phase-stabilized beamtrain with long integration times, allowing high signal-to-noise spectroscopic observations. We have implemented a grism in the secondary camera with a spectral resolution of $R \approx 2000$ to capitalize on this capability.

Once DFPR is commissioned, the next step is astrometry. DFPR measures the delays of stars in both fields. If the baseline separation between the two telescopes, and the instrumental path traversed by light from the two sources, is known then the measured delays can be converted into a separation on the sky. ASTRA aims to deliver 30-100 $\mu$ as astrometric measurement accuracy.

Further description of the ASTRA SPR, DFPR, and astrometric modes can be found in papers by Woillez et al. and Ragland et al. in this volume.
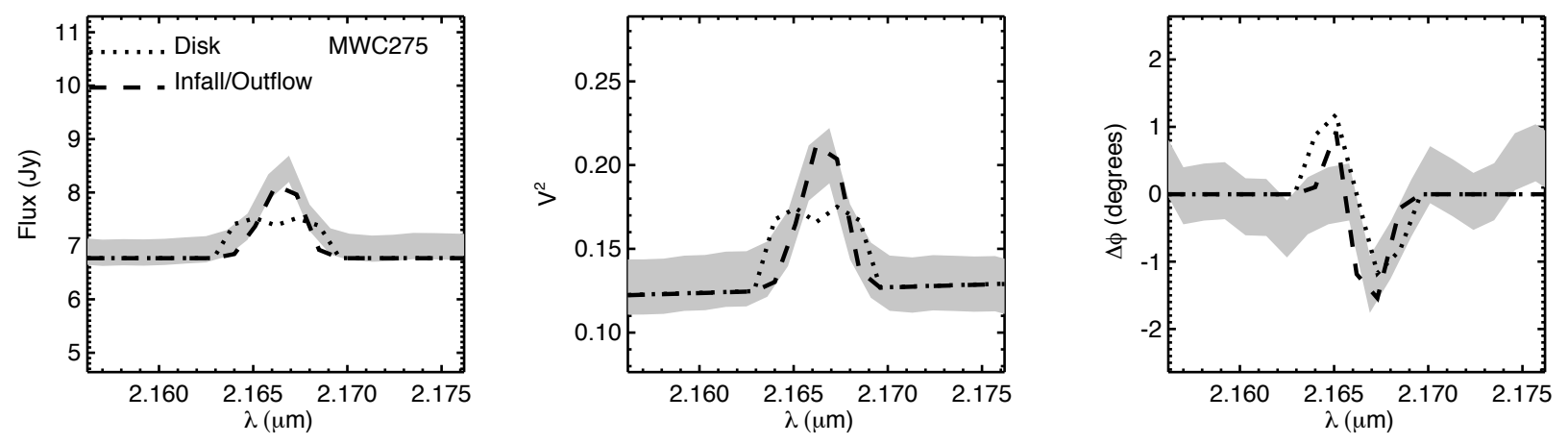

Figure 2. Example of SPR data from KI/ASTRA, with predictions of models over-plotted. The gray regions indicate the 1- $\sigma$ error intervals on the data, after removal of the stellar component. The dotted curves show the predictions of a Keplerian disk model fitted to the data, and the dashed curves show the predictions of a bipolar infall/outflow model. Figure adapted from Eisner et al. 2010. 

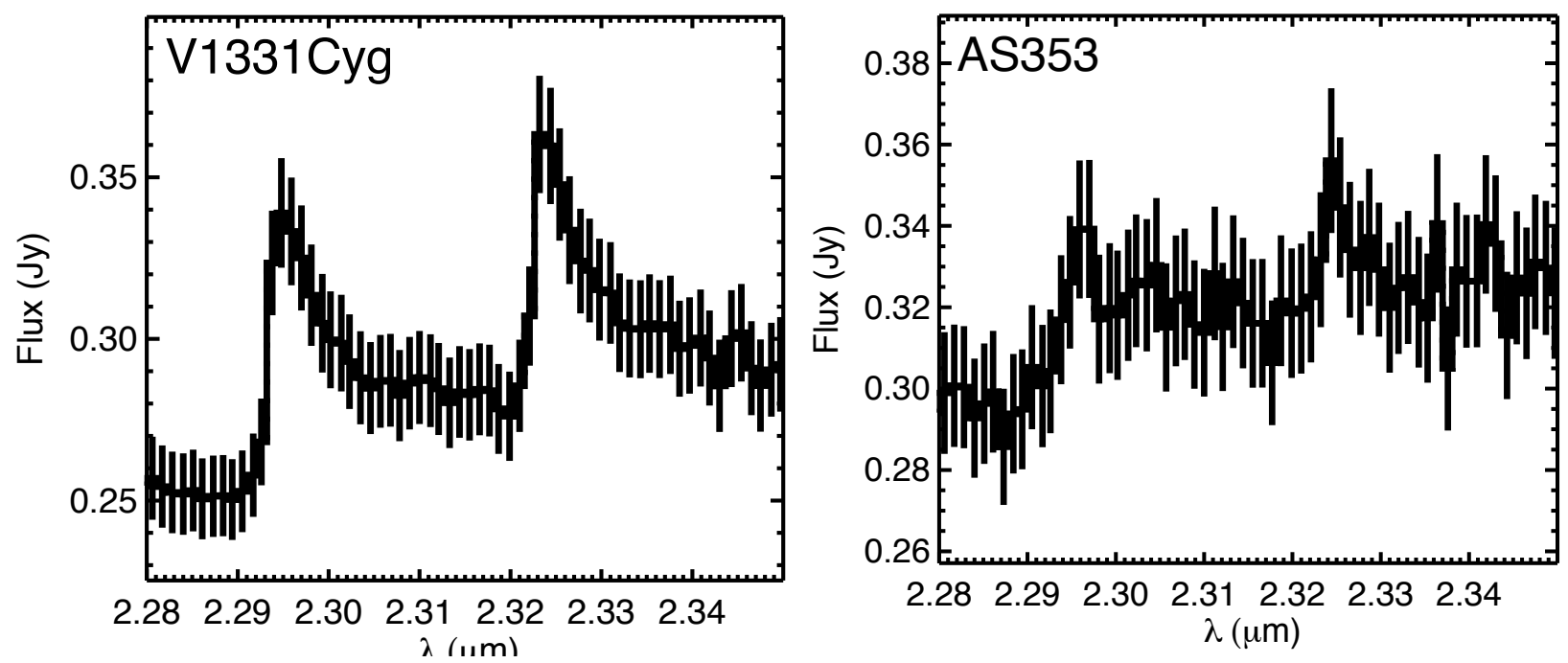

Figure 3. Example spectra of CO overtone bandhead emission in protoplanetary disks, taken with KI/ASTRA. These objects are fainter than the one shown in Figure 2, and so we were unable to observe $V^{2}$ and differential phase signatures with high signal-to-noise across these spectral features. In the future, we plan to employ longer integrations to obtain such measurements.

\section{SPECTROSCOPY OF PROTOPLANETARY DISKS}

Recently, spectrally dispersed interferometry has enabled probes of the gas in protoplanetary disks. ${ }^{1-8}$ Many of these studies focused on $\mathrm{Br} \gamma$ emission, which traces hydrogen gas at $\sim 10,000 \mathrm{~K}$, cascading down to the ground state from highly excited electronic states after recent recombination. Most of these studies found $\operatorname{Br} \gamma$ emission to be more compactly distributed than continuum emission, ${ }^{2,7,8}$ although more extended distributions were seen in a few cases. ${ }^{3,4}$ However, the $\operatorname{Br} \gamma$ emission was only spectrally resolved for a few of these objects, ${ }^{7}$ all of which are bright Herbig Ae/Be stars.

We have used the SPR/grism mode of ASTRA to spectrally and spatially resolve Br $\gamma$ emission from a sample of protoplanetary disks around stars spanning a broad spectral type range. While our observations covered approximately the entire $K$-band, most of our early work focused on the spectral region around the $\operatorname{Br} \gamma$ line of hydrogen at $2.1662 \mu \mathrm{m}$. Measured fluxes, visibilities, and differential phases versus wavelength constrain the spatial distribution and kinematics of the hydrogen gas within $0.1 \mathrm{AU}$ of the central stars in these systems.

The angular resolution of these observations corresponds to stellocentric radii of $\sim 0.1 \mathrm{AU}$ at the distances to our young star targets. However, we used the differential phases observed across the resolved $\operatorname{Br} \gamma$ lines to measure spectro-astrometric offsets of various velocity components. These spatial offsets can be measured with an uncertainty of $<0.01 \mathrm{AU}$.

We found that in many sources, the inferred size of the $\mathrm{Br} \gamma$ emission was $<0.01 \mathrm{AU}$, implying an origin in accretion columns very close to the central star. For other objects, the average size of the $\operatorname{Br} \gamma$ emission was somewhat larger, $\sim 0.1 \mathrm{AU}$. However, analysis of the spectrally resolved data for these objects indicated at least some emission on scales of $0.01 \mathrm{AU}$ or smaller. Thus, we suggest that $\mathrm{Br} \gamma$ emission generally traces accretion, although some $\operatorname{Br} \gamma$ emission may also arise from the inner regions of outflows in some cases. This work is described in detail in Eisner et al. (2010). An example of data, with model fits, is shown in Figure 2.

In the future, we will extend such observations to fainter sources-using longer integrations-and to other spectral regions. In particular, we will probe regions where molecular emission is expected, and map the distribution of molecular material in potentially planet-forming regions (Figure 3). 


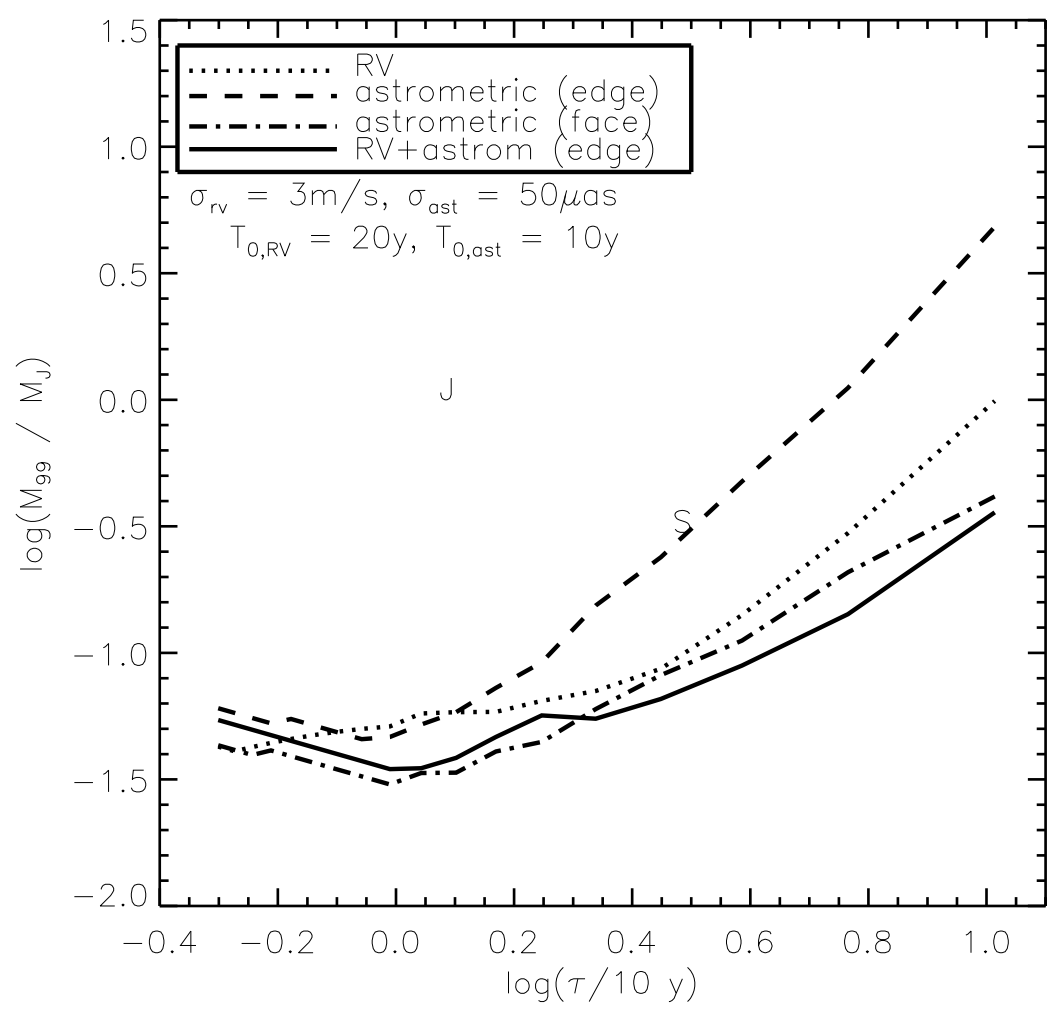

Figure 4. Mass sensitivity (99\% confidence limits) of astrometric, radial velocity, or combined RV+astrometry observations of a star at 10 pc. "J" and "S" indicate the locations of Jupiter and Saturn in this plot. We assume a 20 year RV survey and a 10 year astrometric survey, and measurement precisions of $3 \mathrm{~m} \mathrm{~s}^{-1}$ for the RV data and $50 \mu$ as for the astrometry. In these simulations, measurements are taken once a month. These parameters are chosen to represent current RV surveys and additional data from ASTRA. Edge-on systems only allow 1D astrometry, and so the "edge"-labeled curve is the worst-case. However, even in this case, combining RV and astrometry data allows much better sensitivity than RV alone. Astrometry has a substantial advantage for planets with periods of a few years.

\section{SPATIALLY RESOLVED OBSERVATIONS OF ACTIVE GALACTIC NUCLEI}

Optical and infrared interferometry has historically been limited to bright targets, and so had little relevance for extragalactic astronomy. The enhanced sensitivity enabled by ASTRA's DFPR mode changes this. To date, only the brightest AGNs have been observed with interferometers (KI and VLTI). These observations seem to be consistent with the AGN unification scheme where a molecular torus explains both type 1 and 2 phenomenology. DFPR will enlarge the sample of AGNs for studies of the inner edges of molecular tori. This will help elucidate the geometrical and physical nature of the nuclear obscuration, and the geometrical differences between type 1 and 2 AGNs.

DFPR observations of AGN can also use the grism described above to disperse the AGN light in the phasestabilized channel. The $R \sim 2000$ of the grism is sufficient to spectrally resolve the broad line regions in many AGN, and ASTRA observations can thus provide spectro-astrometric measurements. With $\sim 10 \mu$ as uncertainties on spectro-astrometry with ASTRA (already demonstrated with $\mathrm{SPR}^{9,10}$ ), spatial offsets across the $\operatorname{Br} \gamma$ line in AGN at a distance of $10 \mathrm{Mpc}$ can be detected at the level of $100 \mathrm{AU}$. This is smaller than even the most compact BLR size inferred from reverberation mapping. ${ }^{11}$ ASTRA can measure BLR sizes, and hence directly constrain the masses of the central black holes.

\section{ASTROMETRIC OBSERVATIONS OF EXOPLANETS}

The DFPR mode, in conjunction with astrometry, can be used to measure the wobble of stars induced by orbiting planets. The center-of-mass of a planet/star system is constant, and so a planet induces a star to orbit about the center of mass with an amplitude of

$$
\theta_{\text {ast }}=95 \mu \text { as }\left(\frac{M_{\text {planet }}}{1 \mathrm{M}_{\mathrm{Jup}}}\right)\left(\frac{d}{10 \mathrm{pc}}\right)^{-1}\left(\frac{\tau}{1 \mathrm{yr}}\right)^{2 / 3}\left(\frac{M_{*}}{\mathrm{M}_{\odot}}\right)^{-2 / 3} .
$$

Here, $M_{\text {planet }}$ is the planet mass, $d$ is the distance to the system, $\tau$ is the orbital period of the planet, and $M_{*}$ is the stellar mass. In addition, astrometric measurements will be affected by linear proper motion and parallax 


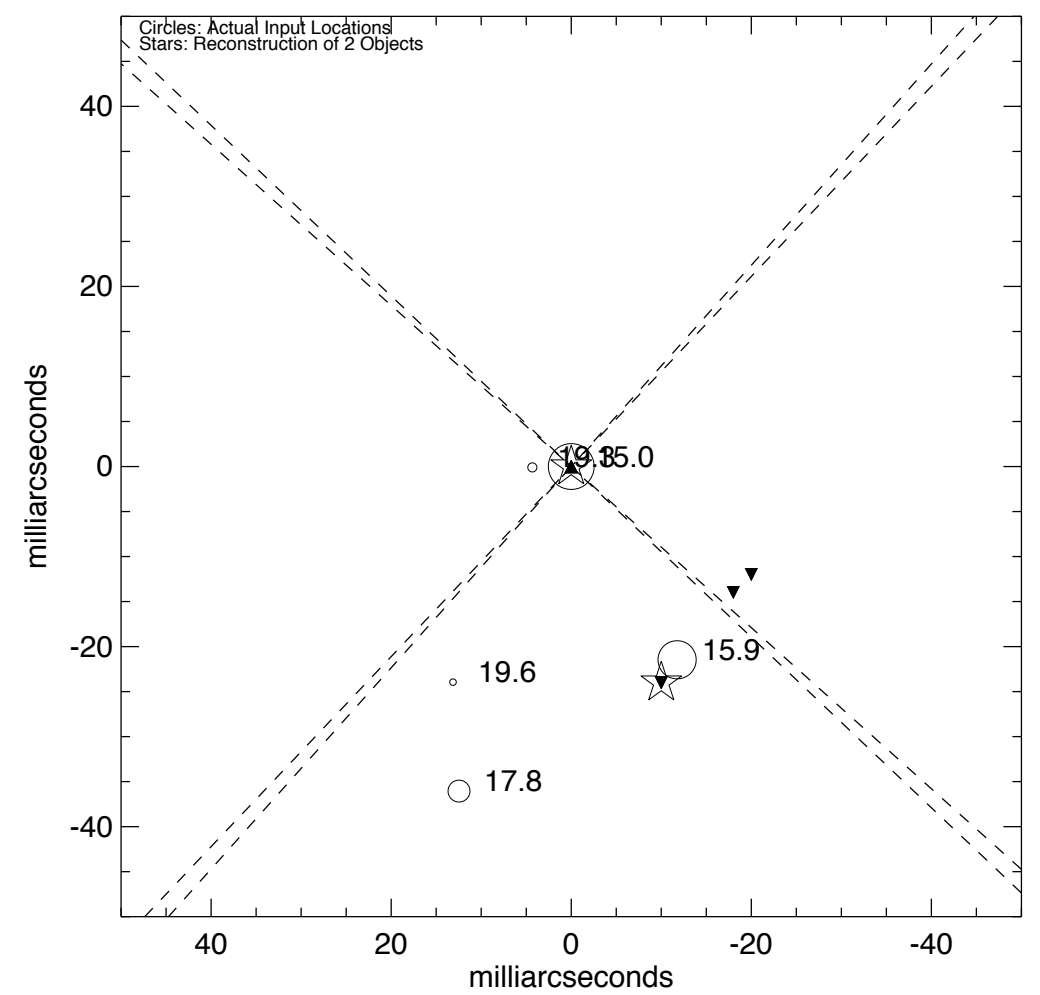

Figure 5. Simulation of the central 50 mas of the Galactic Center. Here we assume SgrA* has a magnitude of $m_{K}=15$ and is located at position $(0,0)$. Several fainter stars, selected based on a luminosity function extrapolated from current observations, were added at random positions. The field was then converted into simulated visibilities, including realistic noise. We fitted a model to the simulated data to try to recover the positions of SgrA* and one other source; i.e., our model only included two objects. Uncertainties on the fitted positions are estimated using bootstrapping. Despite the presence of additional sources in the field, this simple two-source model reproduces the positions of the two brightest sources in the input model to within about a milliarcsecond. Assuming we are able to obtain high signal-to-noise observations, including more sources in our model should improve the fits further.

of the host star. This lowers the effective sensitivity to planets in cases where the orbital period is comparable to or longer than the duration of the astrometric survey. ${ }^{12}$

With measurements sampling a complete orbit with good phase coverage, $50 \mu$ as accuracy is sufficient to detect Jupiter-mass (or lower-mass) planets around nearby stars (Figure 4). Indeed, this level of precision is sufficient to detect planets that have gone undetected by radial velocity surveys (in some cases by combining astrometric and radial velocity data $\left.{ }^{13}\right)$.

Probably the most straightforward application of astrometry is to determine orbital inclinations of exoplanets already known from radial velocity monitoring surveys. With inclination determined, masses can also be determined without a $\sin i$ ambiguity. For multiple planet systems, the inclinations are also important for understanding the stability and provenance of the planets.

Astrometry also has the potential to detect planets that are inaccessible to radial velocity observations. For example, A stars show few spectral lines (at least while on the main-sequence), and so are poor targets for radial velocity surveys. They are, however, fine targets for astrometry. Astrometric monitoring of nearby A stars with KI/ASTRA has the potential to detect massive planets with orbits like that of Jupiter.

A final case that we mention here is planets around pre-main-sequence stars. Starspots can cause significant RV variations at optical wavelengths. ${ }^{14}$ In the near-IR, the flux contrast between the stellar photosphere and the spot is considerably lower, and so starspot noise is less of a problem for near-IR measurements. Furthermore, astrometric amplitudes are typically small for short-period signals, ${ }^{13}$ and so starspot noise with periods near the stellar rotation period are less significant. ${ }^{15}$ ASTRA astrometry is thus well-suited to detecting planets around pre-main-sequence stars.

\section{6. "IMAGING" OF THE GALACTIC CENTER}

With DFPR, ASTRA will achieve sufficient sensitivity to observe stars in short-period orbits about the Galactic Center. Bringing the angular resolution of the Keck Interferometer to bear on these stars enables a number of interesting science cases. 
The simplest is a test for binarity among the population of stars orbiting the galactic center. The KI resolution can constrain binaries with semi-major axes larger than about $10 \mathrm{AU}$. These measurements are important for understanding the origin of the young stars (i.e., the B stars comprising the S cluster) in the Galactic Center.

One of the main science goals of ASTRA is to decrease the confusion of stars in the central regions of the GC, and thereby allow higher precision astrometry. The inner 50 mas around $\mathrm{SgrA}^{*}$ fits into a single AO resolution element, and hence the multiple sources here can not be distinguished with current LGS-AO observations. By imaging this 50-mas-diameter field centered on SgrA* with ASTRA, which provides a resolution element approximately 10 times smaller than AO imaging, we aim to determine the positions of SgrA* and nearby stars.

Any new stars that we can distinguish would have smaller semi-major axes than any of the currently known stars. Assuming a 10 mas semi-major axis, the orbital period would be 0.1 years. It would thus be much simpler to monitor a number of orbits for such stars and to search for precession of perihelia associated with post-Newtonian effects (these are discussed in more detail in the following section). Indeed, simulations have shown that such short-period orbits are optimal for measuring post-Newtonian effects, both because their short periods allow multiple cycles to be observed and because they pass through a deeper part of the potential well around $\mathrm{SgrA}^{*} .{ }^{16}$

In principal, such observations are possible. However, with only a single baseline, our PSF has large sidelobes and we are sensitive to all emission within the 50 mas field of view of KI. Moreover, the KI baseline has limited rotation for the low-declination Galactic Center, and so most of our angular resolution is along a single position angle. To verify that we can, in fact, recover positions of multiple sources within a 50 mas field, we generated simulated observations and fitted multiple-source models to them.

Using a luminosity function extrapolated from the currently known stars in the Galactic Center region, ${ }^{16}$ we estimate the number of stars of a given magnitude within a 50 mas region centered on SgrA*. We typically find that in addition to $\mathrm{SgrA}^{*}$, this field contains one $m_{K} \sim 16$ star, one $m_{K} \sim 18$ star, and stars fainter than $m_{K}=19$. We simulate observations of this field, including photon noise and realistic astrometric noise. Finally, we fit the simulated data with a two-component source model where the positions and fluxes of two stars are free parameters.

Our simulations show that we can recover the position of a $m_{K}=16$ star to a few mas or less, even in the presence of SgrA* and two fainter stars within the 50 mas field of view of KI. Most of the uncertainty is in the direction orthogonal to the position angle of the KI baseline. However, even with $4^{\circ}$ of baseline rotation (which is the maximum we can obtain for observations of the Galactic Center), we can constrain some 2D information. With higher signal-to-noise, our constraints get tighter. Note, too these results are for a simple two-component model. More sophisticated fitting routines, including more than just two components, will likely improve the accuracy with which we can recover source positions.

These observations, while providing astrometric information, do not explicitly require the precise baseline knowledge and monitoring of normal, dual-star astrometric observations (described in the following section). Here stars in a common field are referenced with respect to one another. Thus, these "imaging" observations are, in many ways, simpler than normal astrometry.

\section{ASTROMETRIC MONITORING OF STARS WITH MULTI-YEAR ORBITS ABOUT THE GALACTIC CENTER}

The orbits of stars within 0." 4 of SgrA* have been measured with LGS-AO observations with an accuracy approaching $150 \mu$ as (e.g., Figure 6). However, these precisions are only possible far from SgrA*. Within the central $0 . \prime 1$ or so, confusion limits the measurement accuracy to 0.5 mas or more. For example, the star S0-2, which has a $K$ magnitude of 14 , experiences significant confusion upon closest approach. ${ }^{17}$ This strongly suggests the presence of a star with $m_{K}<16$ within 50 mas of SgrA*. As discussed above, SgrA* itself is most likely confused with this source, as well as with other faint objects in the central 50 mas.

ASTRA aims to provide $\sim 30 \mu$ as astrometric accuracy for stars orbiting around SgrA*.18 ASTRA observations will also be affected by confusion in the inner regions, although to a lesser extent given the higher angular resolution. As for the SgrA* imaging described above, when stars enter confused regions, we will combine our 


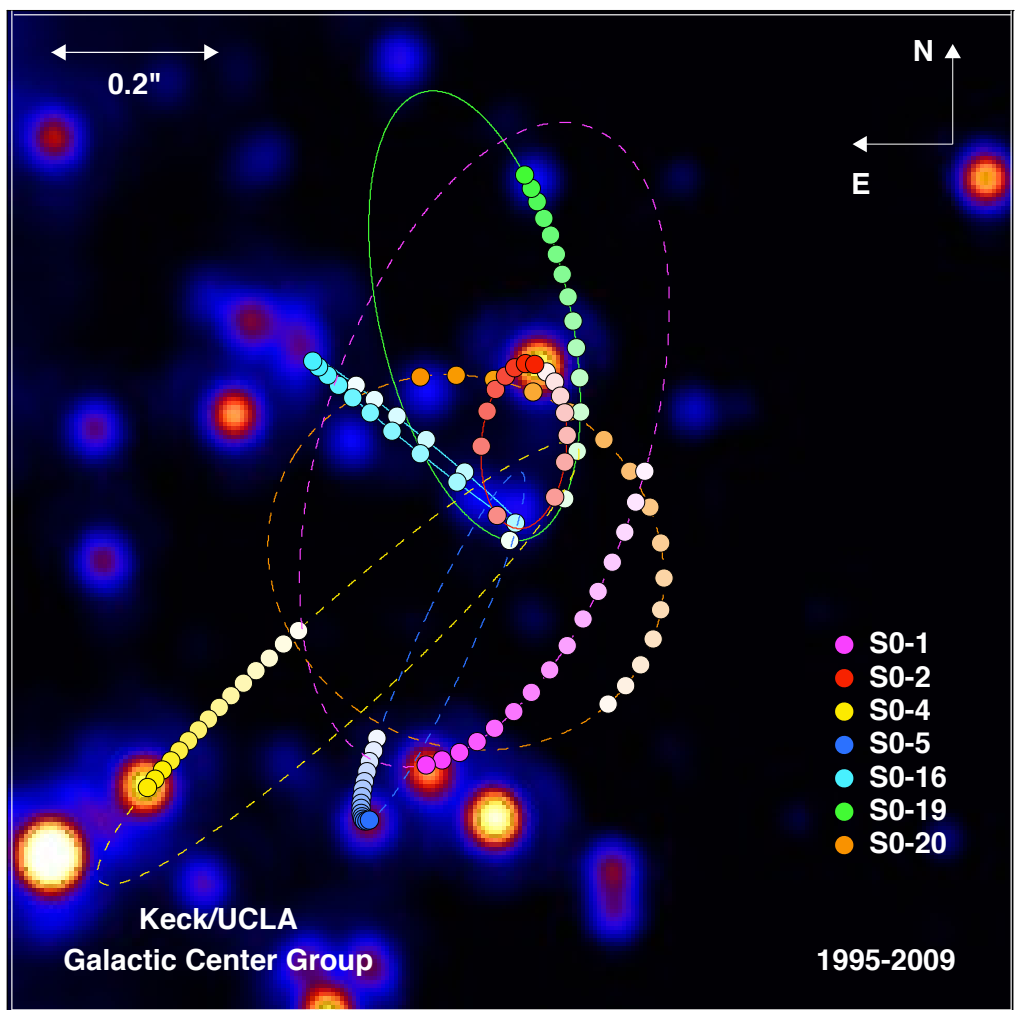

Figure 6. A plot of the orbits of stars in the Galactic Center determined by NGS- and LGS-AO observations. ${ }^{22}$ With KI/ASTRA, we will use the enhanced centroiding and deeper confusion limit afforded by higher angular resolution to improve the accuracy with which these orbits are measured.

astrometric information with imaging information. The astrometry gives the position on the sky of the phase center of our interferometric field of view, while the imaging information provides the position of the star of interest (and potential confusing sources) relative to this phase center position.

The anticipated measurement accuracy of ASTRA is sufficient to constrain post-Newtonian effects, including prograde general relativistic precession and retrograde precession due to the extended (dark) mass distribution near to SgrA*.16 GR effects can be measured even for single orbits of known stars (e.g., S0-2) if we have an astrometric precision of $<100 \mu$ as.

In addition to detecting GR effects on stellar orbits in the GC, ASTRA can measure perturbations to orbits due to the extended matter distribution around the $\mathrm{BH}$, which may consist of a dark matter cusp remaining from the Galaxy formation process. Moreover, scattering of stars by encounters with intermediate mass BHs, which may fall in to the GC under the influence of dynamical friction, would also be detectable with the KI with an astrometric precision of $\sim 100 \mu$ as. Such precision astrometry will also enable measurement of the BH mass and GC distance with an unprecedented 1\% accuracy, crucial for understanding Galactic structure.

Another topic that can be addressed with the instrumentation upgrades proposed here is the origin of the observed flares from the GC. ${ }^{19,20}$ With an astrometric accuracy of tens of $\mu$ as, we can measure the position of the flares relative to other stars in the GC and thereby determine whether this emission arises in an accretion disk at several Schwarzschild radii or in an outflowing jet. If the flares arise in a disk, future astrometric observations with $\mu$ as precision have the potential to observe centroid shifts as flare material executes very tight orbits around the $\mathrm{BH} .{ }^{21}$

\section{ACKNOWLEDGMENTS}

The ASTRA project is made possible by a Major Research Instrumentation (MRI) grant from the National 
Science Foundation (NSF; award \#0619965), and the infrastructure support from the National Aeronautics and Space Administration (NASA) to the Keck Interferometer (KI) project, through the Jet Propulsion Laboratory (JPL) and the NASA Exoplanet Science Institute (NExScI).

\section{REFERENCES}

[1] Eisner, J. A., Chiang, E. I., Lane, B. F., and Akeson, R. L., "Spectrally Dispersed K-Band Interferometric Observations of Herbig Ae/Be Sources: Inner Disk Temperature Profiles," ApJ 657, 347-358 (Mar. 2007).

[2] Eisner, J. A., "Water vapour and hydrogen in the terrestrial-planet-forming region of a protoplanetary disk," Nature 447, 562-564 (May 2007).

[3] Malbet, F., et. al., "Disk and wind interaction in the young stellar object MWC 297 spatially resolved with AMBER/VLTI," A\&A 464, 43-53 (Mar. 2007).

[4] Tatulli, E., et. al., "Constraining the wind launching region in Herbig Ae stars: AMBER/VLTI spectroscopy of HD 104237," A\&A 464, 55-58 (Mar. 2007).

[5] Tatulli, E., Malbet, F., Ménard, F., Gil, C., Testi, L., Natta, A., Kraus, S., Stee, P., and Robbe-Dubois, S., "Spatially resolving the hot CO around the young Be star 51 Ophiuchi," A\&A 489, 1151-1155 (Oct. 2008).

[6] Isella, A., Tatulli, E., Natta, A., and Testi, L., "Gas and dust in the inner disk of the Herbig Ae star MWC 758," A $\dot{E} A$ 483, L13-L16 (May 2008).

[7] Kraus, S., Hofmann, K.-H., Benisty, M., Berger, J.-P., Chesneau, O., Isella, A., Malbet, F., Meilland, A., Nardetto, N., Natta, A., Preibisch, T., Schertl, D., Smith, M., Stee, P., Tatulli, E., Testi, L., and Weigelt, G., "The origin of hydrogen line emission for five Herbig Ae/Be stars spatially resolved by VLTI/AMBER spectro-interferometry," A\&A 489, 1157-1173 (Oct. 2008).

[8] Eisner, J. A., Graham, J. R., Akeson, R. L., and Najita, J., "Spatially Resolved Spectroscopy of Sub-AUSized Regions of T Tauri and Herbig Ae/Be Disks," ApJ 692, 309-323 (Feb. 2009).

[9] Pott, J.-U., et al., "Probing local density inhomogeneities in a circum-Be-star disk using the new spectroastrometry at the Keck Interferometer," ApJ, submitted

[10] Eisner, J. A., et al., "Spatially and Spectrally Resolved Hydrogen Gas within 0.1 AU of T Tauri and Herbig Ae/Be Stars," ApJ, submitted

[11] Bentz, M. C., Peterson, B. M., Pogge, R. W., Vestergaard, M., and Onken, C. A., "The Radius-Luminosity Relationship for Active Galactic Nuclei: The Effect of Host-Galaxy Starlight on Luminosity Measurements," ApJ 644, 133-142 (June 2006).

[12] Eisner, J. A. and Kulkarni, S. R., "Sensitivity of the Astrometric Technique in Detecting Outer Planets," ApJ 561, 1107-1115 (Nov. 2001).

[13] Eisner, J. A. and Kulkarni, S. R., "Detecting Outer Planets in Edge-on Orbits: Combining Radial Velocity and Astrometric Techniques," ApJ 574, 426-429 (July 2002).

[14] Desort, M., Lagrange, A., Galland, F., Udry, S., and Mayor, M., "Search for exoplanets with the radialvelocity technique: quantitative diagnostics of stellar activity," AEA 473, 983-993 (Oct. 2007).

[15] Makarov, V. V., Beichman, C. A., Catanzarite, J. H., Fischer, D. A., Lebreton, J., Malbet, F., and Shao, M., "Starspot Jitter in Photometry, Astrometry, and Radial Velocity Measurements," ApJ 707, L73-L76 (Dec. 2009).

[16] Weinberg, N. N., Milosavljević, M., and Ghez, A. M., "Stellar Dynamics at the Galactic Center with an Extremely Large Telescope," ApJ 622, 878-891 (Apr. 2005).

[17] Gillessen, S., Eisenhauer, F., Fritz, T. K., Bartko, H., Dodds-Eden, K., Pfuhl, O., Ott, T., and Genzel, R., "The Orbit of the Star S2 Around SGR A* from Very Large Telescope and Keck Data," ApJ 707, L114-L117 (Dec. 2009).

[18] Pott, J.-U., Woillez, J., Wizinowich, P. L., Eckart, A., Glindemann, A., Ghez, A. M., \& Graham, J. R., "First spectro-interferometry on galactic center sources in the infrared: results and science prospects at the sensitivity limit of current larger aperture arrays," Proc. SPIE, 7013, 61 (2008).

[19] Genzel, R., Schödel, R., Ott, T., Eckart, A., Alexander, T., Lacombe, F., Rouan, D., and Aschenbach, B., "Near-infrared flares from accreting gas around the supermassive black hole at the Galactic Centre," Nature 425, 934-937 (Oct. 2003). 
[20] Ghez, A. M., Wright, S. A., Matthews, K., Thompson, D., Le Mignant, D., Tanner, A., Hornstein, S. D., Morris, M., Becklin, E. E., and Soifer, B. T., "Variable Infrared Emission from the Supermassive Black Hole at the Center of the Milky Way," ApJ 601, L159-L162 (Feb. 2004).

[21] Broderick, A. E. and Loeb, A., "Imaging bright-spots in the accretion flow near the black hole horizon of Sgr A*," MNRAS 363, 353-362 (Oct. 2005).

[22] Ghez, A. M., Salim, S., Weinberg, N. N., Lu, J. R., Do, T., Dunn, J. K., Matthews, K., Morris, M. R., Yelda, S., Becklin, E. E., Kremenek, T., Milosavljevic, M., and Naiman, J., "Measuring Distance and Properties of the Milky Way's Central Supermassive Black Hole with Stellar Orbits," ApJ 689, 1044-1062 (Dec. 2008). 